\title{
Let's Get Virtual: Examination of Best Practices to Provide Public Access to Digital Versions of Three-Dimensional Objects
}

Tanya M. Johnson

\begin{abstract}
Three-dimensional objects are important sources of information that should not be ignored in the increasing trend towards digitization. Previous research has not addressed the evaluation of digitized versions of three-dimensional objects. This paper first reviews research concerning such digitization, in both two and three dimensions, as well as public access in this context. Next, evaluation criteria for websites incorporating digital versions of three-dimensional objects are extrapolated from previous research. Finally, five websites are evaluated, and suggestions for best practices to provide public access to digital versions of three-dimensional objects are proposed.
\end{abstract}

\section{INTRODUCTION}

Much of the literature surrounding the increased efforts of libraries and museums to digitize content has focused on two-dimensional forms, such as books, photographs, or paintings. However, information does not only come in two dimensions; there are sculptures, artifacts, and other three-dimensional objects that have been unfortunately neglected by this digital revolution. As one author stated, "While researchers do not refer to three-dimensional objects as commonly as books, manuscripts, and journal articles, they are still important sources of information and should not be taken for granted" (Jarrell 1998, 32).

The importance of three-dimensional objects as information that can and should be shared is not a new phenomenon; indeed, as early as 1887, museologists and educators forwarded the view that "museums were in effect libraries of objects" that provided information not supplied by books alone (Given and McTavish 2010,11). However, it is only recently, with the advent of newer technological mechanisms, that such objects could be shared with the public on a larger scale. No longer do people need to physically visit museums to experience and learn from three-

dimensional objects. Rather, various techniques have been utilized to place digital versions of such objects on the websites of museums and archives, and projects have been created by various universities in order to enhance that digital experience. Nevertheless, as Newell (2012) states:

Collections-holding institutions increasingly regard digital resources as additional objects of significance, not as complete replacements for the original. Digital technologies work best when they enable people who feel connected to museum objects to have the freedom to deepen these

Tanya M. Johnson (tmjohnso@gmail.com), a recent MLIS degree graduate from the School of Communication \& Information, Rutgers, The State University of New Jersey, is winner of the 2016 LITA/Ex Libris Student Writing Award. 
relationships and, where appropriate, to extend outsiders' understandings of

the objects' cultural contexts. The raison d'être of museums and other cultural institutions remains centred on the primacy of the object and in this sense continues to privilege material authenticity. (303)

In this regard, three-dimensional visualization of physical objects can be seen as the next step for museums and cultural heritage institutions that seek to further patrons' connection to such objects via the internet.

Indeed, in this digital age, the goals of museums and archives are changing, converging with those of libraries to focus more efforts on providing information to the public, and, along with the growing trend to digitize information contained within libraries, there has been a concomitant trend to digitize the contents of museums in order to provide greater public access to collections (Given and McTavish 2010). In light of this progress, this paper will review various methods of presenting three-dimensional objects to the public on the internet and, based on an evaluation of five digital collections, attempt to provide some advice as to best practices for museums or institutions seeking to digitize such objects and present them to the public via a digital collection.

\section{LITERATURE REVIEW}

\section{Two-Dimensional Digitization}

There are many ways to present digital versions of three-dimensional objects on a webpage, ranging from simple two-dimensional photography to complicated three-dimensional scanning and rendering. Beginning on the simpler end of the scale, Bincsik, Maezaki, and Hattori (2012) describe the process of photographing Japanese decorative art objects in order to create an image database of objects from multiple museums. Specifically, the researchers explain that they need high quality photographs showing each object in all directions, as well as close-up images of fine details, in order to recreate the physical research experience as closely as possible. They also note that, for the same reason, the context of each object must be recorded, including photographs of any wrapping or storage materials and accompanying documentation. For this project, the researchers utilized Nikon professional or semi-professional cameras, with zoom and macro lenses, and often used small apertures to increase depth-of-field. At times, they also took measurements of the objects in order to assist museums in maintaining accurate records. The raw image files were then processed with programs such as Adobe Photoshop, saved as original TIF files, and converted into JPEG format for upload. Despite the success of the project, the researchers also noted the limitations of digitizing three-dimensional objects:

With decorative art objects some information is inevitably lost, such as the weight of the object, the feeling of its surface texture or the sense of its functionality in terms of proportions and balance. Digital images clearly can fulfill many research objectives, but in some cases they can only be used as references. One objective of the decorative arts database is to advise the researcher in selecting which objects should be examined in person. (Bincsik, Maezaki, and Hattori 2012, 46)

One difficulty with photography, particularly when digitizing artwork, is that color is a function of light. Thus, a single object will often appear to be different colors when photographed in different lighting conditions using conventional digital cameras, which process images using RGB filters. 
More accurate representations of objects can be acquired using multispectral imaging, which uses a higher number of parameters (the international standard is 31, compared to RGB's 3) in order to obtain more information about the reflectance of an object at any particular point in space (Novati, Pellegri, and Schettini 2005). Multispectral imaging, however, is very expensive and, despite some researchers' attempts to create affordable systems (e.g., Novati, Pellegri, and Schettini 2005), the acquisition of multispectral images is generally limited to large institutions with considerable funding (Chane et al. 2013).

The use of two-dimensional photography to digitize objects is not limited to the arts; in the natural sciences, different types of photographic equipment have been developed to document existing collections and enhance scientific observation. Gigapixel imaging, for example, has been utilized to allow museum visitors to virtually explore large petroglyphs located in remote locations as well as for documentation and viewing of dinosaur bone specimens that are not on public display (Louw and Crowley 2013). This technology consists of taking many, very high resolution photographs that are then, via computer software, "aligned, blended, and stitched" together to create one extremely detailed composite image (Louw and Crowley 2013, 89-90). Robotic systems, such as GigaPan, have been developed to speed up the process and permit rapid recording and processing of the necessary area. Once the gigapixel image is created, it can then be uploaded and displayed on the web in dynamic form, including spatial navigation of the image with embedded text, audio, or video at specific locations and zoom levels to provide further information (Louw and Crowley 2013).

Various types of gigapixel imaging, including the GigaPan system, have also been used to digitize important collections of biological specimens, particularly insects, which are often stored in large drawers. One study examined the documentation of entomological specimens by "whole-drawer imaging" using various gigapixel imaging technologies (Holovachov, Zatushevsky, and Shydlovsky 2014). The researchers explained that different gigapixel imaging systems (many of which are commercial and proprietary) utilize different types of cameras and lenses, as well as different types of software for processing. However, despite the expensive cost of some commercially available systems, it is possible for museums and other institutions to create their own, economically viable versions. The system created by Holovachov, Zatushevsky, and Shydlovsky utilized a standard SLR camera, fitted with a macro lens and attached to an immovable stand. The researchers manually set up lighting, focus, aperture, and other settings, and moved the insect drawer along a pre-determined grid pattern in order to obtain the multiple overlapping photographs necessary to create a large gigapixel image. They used a freely available stitching software program and manually corrected stitching artifacts and color balance issues that resulted from the use of a non-telecentric lens. ${ }^{1}$ Despite the lower cost of their individualized system, however, the researchers noted that the process was much more time-consuming and necessitated more labor from workers digitizing the collection. Moreover, technologically speaking, the researchers emphasized the limits of two-dimensional imaging, given that the

\footnotetext{
1The difference between telecentric and non-telecentric lenses is explained by the researchers:

"Contrary to ordinary photographic lenses, object-space telecentric lenses provide the same object magnification at all possible focusing distances. An object that is too close or too far from the focus plane and not in focus, will be the same size as if it were in focus. There is no perspective error and the image projection is parallel. Therefore, when such a lens is used to take images of pinned insects in a box, all vertical pins will appear strictly vertical, independent of their position within the camera's field of view" (Holovachov, Zatushevsky, and Shydlovsky 2014, 7).
} 
"diagnostic characteristics of three-dimensional insects," as well as the accompanying labels, are often invisible when a drawer is only photographed from the top. Thus, the researchers concluded that, ultimately, "the whole-drawer digitizing of insect collections needs to be transformed from two-dimensions to three-dimensions by employing complex imaging techniques (simultaneous use of multiple cameras positioned at different angles) and a digital workflow" (Holovachov, Zatushevsky, and Shydlovsky 2014, 7).

\section{Three-Dimensional Digitization}

Given the goal of obtaining as accurate a representation as possible when digitizing objects, many researchers have turned to the use of various techniques in order to obtain three-dimensional data. Acquiring a three-dimensional image of an object takes place in three steps:

1. Preparation, during which certain preliminary activities take place that involve the decision about the technique and methodology to be adopted as well as the place of digitization, security planning issues, etc.

2. Digital recording, which is the main digitization process according to the plan from phase 1.

3. Data processing, which involves the modeling of the digitized object through the unification of partial scans, geometric data processing, texture data processing, texture mapping, etc. (Pavlidis et al. 2007, 94)

Steps 2 and 3 have been more technically described as (2) obtaining data from an object to create point clouds (from thousands to billions of $\mathrm{X}, \mathrm{Y}, \mathrm{Z}$ coordinates representing loci on the object); and (3) processing point clouds into polygon models (creating a surface on top of the points), which can then be mapped with textures and colors (Metallo and Rossi 2011).

There are several techniques that can be utilized to acquire three-dimensional data from a physical object. Table 1 explains the four general methods most commonly used by museums. 


\begin{tabular}{|c|c|c|c|c|}
\hline Type & Description & Positives & Negatives & $\begin{array}{l}\text { Approx. } \\
\text { Price Range }\end{array}$ \\
\hline Laser Scanning & $\begin{array}{l}\text { A laser source emits light } \\
\text { onto the object's surface, } \\
\text { which is detected by a digital } \\
\text { camera; geometry of the } \\
\text { object is extracted by } \\
\text { triangulation or time of flight } \\
\text { calculations }\end{array}$ & $\begin{array}{l}\text { High accuracy in } \\
\text { capturing } \\
\text { geometry; can } \\
\text { capture small } \\
\text { objects and entire } \\
\text { buildings (using } \\
\text { different hardware) }\end{array}$ & $\begin{array}{l}\text { Limited texture } \\
\text { and color } \\
\text { captured; shiny } \\
\text { surfaces refract } \\
\text { the laser }\end{array}$ & $\begin{array}{l}\$ 3,000- \\
\$ 200,000\end{array}$ \\
\hline $\begin{array}{l}\text { White Light } \\
\text { (Structured Light) } \\
\text { Scanning }\end{array}$ & $\begin{array}{l}\text { A pattern of light is projected } \\
\text { onto the object's surface, and } \\
\text { deformations in that pattern } \\
\text { are detected by a digital } \\
\text { camera; geometry is } \\
\text { extracted by triangulation } \\
\text { from deformations }\end{array}$ & $\begin{array}{l}\text { Captures texture } \\
\text { details, making it } \\
\text { very accurate; can } \\
\text { capture color }\end{array}$ & $\begin{array}{l}\text { Dark, shiny, or } \\
\text { translucent } \\
\text { objects are } \\
\text { problematic }\end{array}$ & $\begin{array}{l}\$ 15,000- \\
\$ 250,000\end{array}$ \\
\hline Photogrammetry & $\begin{array}{l}\text { Three-dimensional data is } \\
\text { extracted from multiple two- } \\
\text { dimensional pictures }\end{array}$ & $\begin{array}{l}\text { Can capture small } \\
\text { objects and } \\
\text { mountain ranges; } \\
\text { good color } \\
\text { information }\end{array}$ & $\begin{array}{l}\text { Need either } \\
\text { precise } \\
\text { placement of } \\
\text { cameras or more } \\
\text { precise software } \\
\text { to obtain } \\
\text { accurate data }\end{array}$ & $\begin{array}{l}\text { Cameras: } \\
\$ 500- \\
\$ 50,000 \\
\text { Software: } \\
\text { free- } \\
\$ 40,000\end{array}$ \\
\hline $\begin{array}{l}\text { Volumetric } \\
\text { Scanning }\end{array}$ & $\begin{array}{l}\text { Magnetic resonance imaging } \\
\text { (MRI) uses a strong magnetic } \\
\text { field and radio waves to } \\
\text { detect geometric, density, } \\
\text { volume and location } \\
\text { information; computed } \\
\text { tomography (CT) uses } \\
\text { rotating x-rays to create two- } \\
\text { dimensional slices, which can } \\
\text { then be reconstructed into } \\
\text { three-dimensional images }\end{array}$ & $\begin{array}{l}\text { Both types can view } \\
\text { the interior and } \\
\text { exterior of an } \\
\text { object; CT can be } \\
\text { used for reflective } \\
\text { or translucent } \\
\text { objects; MRI can } \\
\text { image soft tissues }\end{array}$ & $\begin{array}{l}\text { No color } \\
\text { information; MRI } \\
\text { requires object to } \\
\text { have high water } \\
\text { content }\end{array}$ & $\begin{array}{l}\$ 200,000- \\
\$ 2,000,000\end{array}$ \\
\hline
\end{tabular}

Table 1. Description of four general methods of acquiring three-dimensional data about physical objects (table information compiled by reference to Pavlidis et al. 2007; Metallo and Rossi 2011; Abel et al. 2011; and Berquist et al. 2012).

The type of three-dimensional digitization used can ultimately depend upon the types of objects to be imaged or the type of data needed. For example, in digitizing human skeletal collections, one study explained that three-dimensional laser scanning was an advantageous technique to create models of bones for preservation and analysis, but cautioned that CT scans would be needed to examine the internal structures of such specimens (Kuzminsky and Gardiner 2012). Another study 
utilized several techniques in an attempt to decipher graffiti inscriptions on ancient Roman pottery shards, ultimately concluding that high-resolution photography (similar to gigapixel imaging) and three-dimensional laser scanning both provided detailed and helpful data (Montani et al. 2012). Additionally, sometimes multiple types of digitization can be used for the same objects with similar results. One study, for example, obtained virtually equivalent threedimensional models of the same object using laser scanning and two types of photogrammetry (Lerma and Muir 2014).

Most recently, researchers have been utilizing combinations of digitization techniques to obtain the most accurate representations possible. Chane et al. (2013), for example, examined methods of combining three-dimensional digitization with multispectral photography in order to obtain enhanced information concerning the physical object in question. The researchers explained that combining the two processes is difficult because, in order to obtain multispectral textural data that is mapped to geometric positions, the object must be imaged from identical locations by multiple scanners/cameras or else the data processing that combines the two types of data becomes extremely complex. As a compromise, the researchers created a system of optical tracking based on photogrammetry techniques that permits the collection and integration of geometric positioning data and multispectral textures utilizing precise targeting procedures. However, the researchers noted that most systems integrating multispectral photography with threedimensional digitization tended to be quite bulky, did not adapt easily to different types of objects, and needed better processing algorithms for more complex three-dimensional objects (Chane et al. 2013).

\section{Public Access to Three-Dimensionally Digitized Objects}

Despite museums' growing focus on increasing public access to collections via digitization (Given and McTavish 2010), there is very little literature addressing public access to three-dimensionally digitized objects. Indeed, studies in this realm tend to focus on the technological aspects of either the modeling of specific objects or collections or website viewing of three-dimensional models. For example, Abate et al. (2011) described the three-dimensional digitization of a particular statue from the scanning process to its ultimate depiction on a website. The researchers explained in detail the particular software architecture utilized in order to permit the remote rendering of the three-dimensional model on users' computers via a Java applet without compromising quality or necessitating download of potentially copyrighted works. By contrast, literature concerning the Digital Michelangelo project, during which researchers three-dimensionally digitized various Michelangelo works, focused on the method used to create an accurate three-dimensional model, complete with color and texture mapping, and a visualization tool (Dellepiane et al. 2008).

One study did describe a project that was designed to place three-dimensional data about various cultural artifacts in an online repository for curators and other professionals (Hess et al. 2011). This repository was contained within database management software, a web-based interface was designed for searching, and user access to three-dimensional images and models was provided via an ActiveX plugin. Despite the potential of the prototype, however, it appears that the project has ceased, ${ }^{2}$ and the institution's current three-dimensional imaging project is focused on the design

${ }^{2}$ See http://www.ucl.ac.uk/museums/petrie/research/research-projects/3dpetrie/3d projects/3d-projects-past/ecurator. 
of a traveling exhibition incorporating, among other things, three-dimensional models of artifacts and physical replicas created from such models. ${ }^{3}$

Studies that do address public access directly tend to focus on the improvement of museum websites generally. For example, in terms of user expectations of museum websites, one study found that approximately 63 percent of visitors to a museum's website did so in order to search the digital collection (Kravchyna and Hastings 2002). Another study found four types of museum website users, who each had different needs and expectations of sites. Relevantly, educators sought collections that were "the more realistic the better," including suggestions like incorporating three-dimensional simulations of physical objects so that students could "explore the form, construction, texture and use of objects" (Cameron 2003, 335). Further, non-specialist users "value free choice learning" and "access online collections to explore and discover new things and build on their knowledge base as a form of entertainment" (Cameron 2003, 335).

Similarly, some studies have addressed the incorporation of Web 2.0 technologies into museum websites. Srinivasan et al. (2009), for example, argue that Web 2.0 technologies must be integrated into museum catalogs rather than simply layered over existing records because users' interest in objects is increased by participation in the descriptive practice. An implementation of this concept is found in Hunter and Gerber's (2010) system of social tagging attached to threedimensional models.

This paper is an effort to address the gap between the technical process of digitizing and presenting three-dimensional objects on the web and the user experience of such. Through the evaluation of five websites, this paper will provide some guidance for the digitization of threedimensional objects and their presentation in digital collections for public access.

\section{METHODOLOGY AND EVALUATIVE CRITERIA}

Evaluations of digital museums are not as prevalent as evaluations of digital libraries. However, given the similar purposes of digital museums and digital libraries, it is appropriate to utilize similar criteria.

For digital libraries, Saracevic (2000) synthesized evaluation criteria into performance questions in two broad areas: (a) user-centered questions, including how well the digital library supports the society or community served, how well it supports institutional or organizational goals, how well it supports individual users' information needs, and how well the digital library's interface provides access and interaction; and (b) system- centered questions, including hardware and network performance, processing and algorithm performance, and how well the content of the collection is selected, represented, organized, and managed. Xie (2008) focused on user-centered evaluation and found five general criteria that exemplified users' own evaluations of digital libraries: interface usability, collection quality, service quality, system performance, and user satisfaction. Parandjuk (2010) used information architecture to construct criteria for the evaluation of a digital library, including the following:

- uniformity of standards, including consistency among webpages and individual records;

- findability, including ease of use and multiple ways to access the same information;

- sub-navigation, including indexes, sitemaps, and guides;

\footnotetext{
${ }^{3}$ See http://www.3dencounters.com.
} 
- contextual navigation, including simplified searching and co-location of different types of resources;

- language, including consistency in labeling across pages and records and appropriateness for the audience; and

- integration of searching and browsing.

This system is particularly appropriate in the context of digital museums, as it emphasizes the curatorial or organizational aspect of the collection in order to support learning objectives.

In one comprehensive evaluation of the websites of art museums, Pallas and Economides (2008) created a framework for such evaluation, incorporating six dimensions: content, presentation, usability, interactivity and feedback, e-services, and technical. Each dimension then contained several specific criteria. Many of the criteria overlapped, however, and three-dimensional imaging, for example, was placed within the e-services dimension, under virtual tours, although it could have been placed within presentation, with other multimedia criteria, or even within interactivity, with interactive multimedia applications.

The problem in trying to evaluate a particular part of a museum's website, namely, the way it presents three-dimensional objects in digital form, is that the level of specificity almost renders many of the evaluation criteria from previous studies irrelevant. As Hariri and Norouzi (2011) suggest, evaluation criteria should be based on the objective of the evaluation. Hence, based on portions of the above-referenced studies, this author has created a more focused evaluation framework, concentrating on criteria that are particularly relevant to museums' digital presentations of three-dimensional objects. This framework is detailed in table 2, below.

\begin{tabular}{ll}
\hline Dimension & Description \\
\hline Functionality & $\begin{array}{l}\text { What technology is used to display the object? How well does it work? } \\
\text { Must programs or files be downloaded? Are the loading times of displays } \\
\text { acceptable? }\end{array}$ \\
Usability & $\begin{array}{l}\text { How easy is the site to use? What is the navigation system? Are there } \\
\text { searching and browsing functions, and how well does each work? How } \\
\text { findable are individual objects? }\end{array}$ \\
Presentation & $\begin{array}{l}\text { How does the display of the object look? What is the context in which the } \\
\text { object is presented? Are there multiple viewing options? Is there any } \\
\text { interactivity permitted? }\end{array}$ \\
Content & $\begin{array}{l}\text { Does the site provide an adequate collection of objects? For individual } \\
\text { objects, is there sufficient information provided? Is there additional } \\
\text { educational content? }\end{array}$ \\
\hline
\end{tabular}

Table 2. Summary of evaluative criteria

Five digital collections, specified below, will be evaluated based on these criteria. This will be done in a case study manner, describing each website based on the above criteria and then using those evaluations to make suggestions for best practices.

\section{RESULTS}


It is difficult to compare different types of digital collections, particularly when the focus is on different types of technology utilized to display similar objects. However, because the goal here is to determine the best practices for the digital presentation of three-dimensional objects, it is important to evaluate a variety of techniques in a variety of fields. Thus, the following digital collections have been chosen to illustrate different ways in which such objects can be displayed on a website.

\section{Museum of Fine Arts, Boston (MFA) (http://www.mfa.org/collections)}

The MFA, both in person and online, boasts a comprehensive and extensive collection of art and historical artifacts of varying forms. The website is very easy to navigate, with well-defined browsing options and easy search capabilities, allowing for refinement of results by collection or type of item. There are many collections, which are well organized and curated into separate exhibits and galleries. In addition, when viewing each gallery, suggestions are linked for related online exhibitions as well as tours and exhibits at the physical museum. Each item record contains a detailed description of the item as well as its provenance. Thus, the MFA website attains a very high rating for usability and content. However, individual items are represented by only single pictures of varying quality. Some pictures are color, some are black and white, and no two pictures appear to have the same lighting. Additionally, despite being slow to load, even the pictures that appear to be of the best quality cannot be of high resolution, as zooming in makes them slightly blurry. Accordingly, the MFA website receives a medium rating for functionality and a low rating for presentation.

\section{Digital Fish Library (DFL) (http://www.digitalfishlibrary.org/index.php)}

The DFL project is a comprehensive program that utilizes MRI scanning to digitize preserved biological fish samples from a particular collection housed at the Scripps Institution of Oceanography. After MRI scans of a specimen are taken, the data is processed and translated into various views that are placed on the website, accompanied by information about each species (Berquist et al. 2012).

Navigating the DFL website is very intuitive, as the individual specimen records are organized by taxonomy. It is easy to search for particular species or browse through the clickable, pictorial interface. Records for each species include detailed information about the individual specimen, the specifics of the scans used to image each, and broader information about the species. Individual records also provide links to other species within the taxonomic family. Thus, the DFL website attains high ratings in both usability and content. For functionality and presentation, however, the ratings are medium. Although for each item there are videos and still images obtained from threedimensional volume renderings and MRI scans, they are small in size and have low resolution. There is no interactive component, with the possible exception of the "digital fish viewer" that supposedly requires Java, but this author could not get it to work despite best efforts. One nice feature, shown in figure 1 below, is that some of the specimen records have three-dimensional renderings showing and explaining the internal structures of the species. 


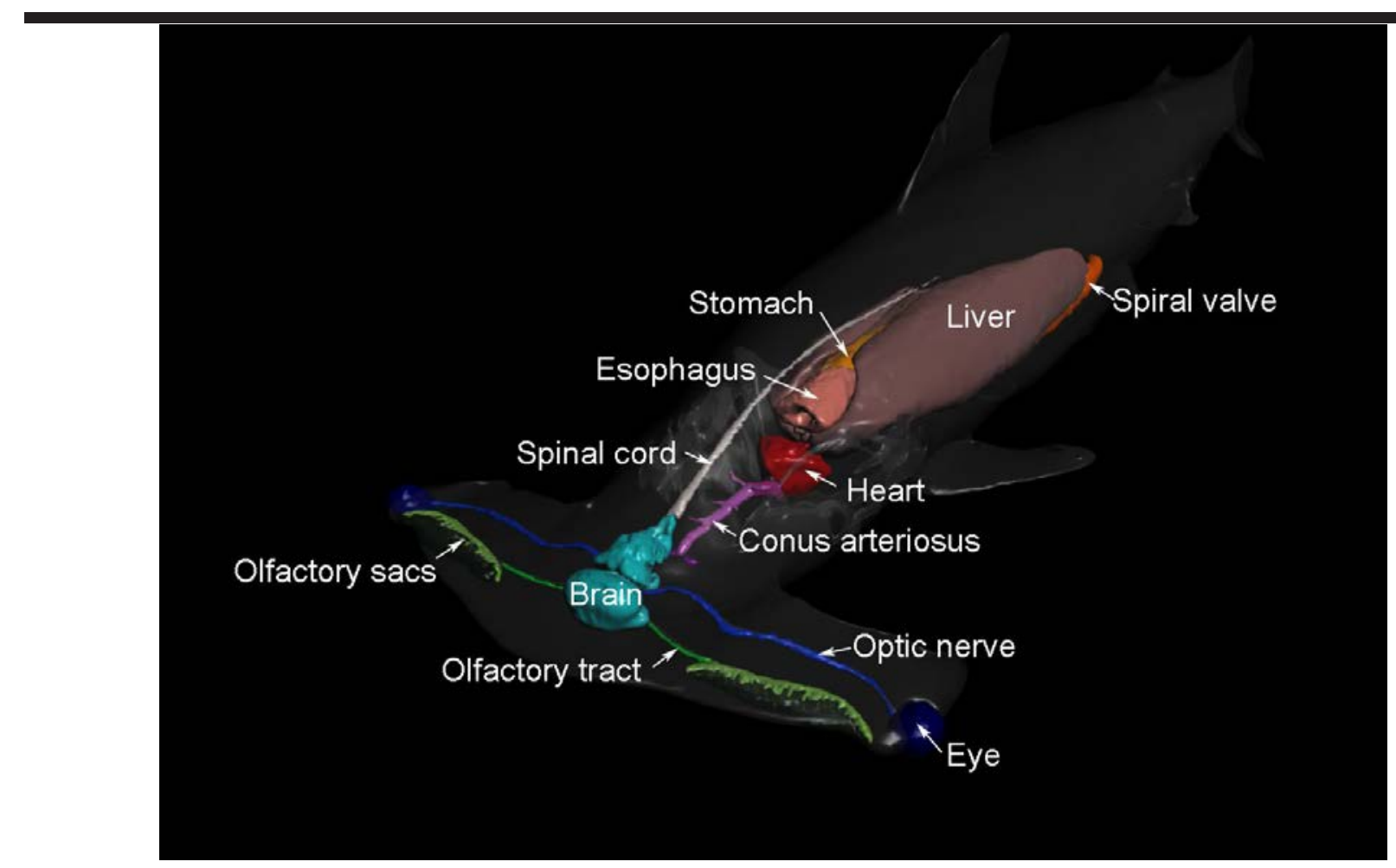

Figure 1. Annotated three-dimensional rendering of internal structures of hammerhead shark, from the Digital Fish Library (http://www.digitalfishlibrary.org/library/ViewImage.php?id=2851)

\section{The Eton Myers Collection (http://etonmyers.bham.ac.uk/3D-models.html)}

The Eton Myers Collection of ancient Egyptian art is housed at Eton College, and a project to threedimensionally digitize the items for public access was undertaken via collaboration between that institution and the University of Birmingham. Digitization was accomplished with threedimensional laser scanners, data was then processed with Geomagic software to produce point cloud and mesh forms, and individual datasets were reduced in size and converted into an appropriate file type to allow for public access (Chapman, Gaffney, and Moulden 2010).

Usability of the Eton Myers Collection website is extremely low. The initial interface is simply a list of three-dimensional models by item number with a description of how to download the appropriate program and files. Another website from the University of Birmingham (http://mimsy.bham.ac.uk/info.php? $\mathrm{f}=$ option8\&type=browse\&t=objects\&s=The+Eton+Myers+Col lection) contains a more museum-like interface, but contains many more records for objects than are contained on the initial list of three-dimensional models. Moreover, most of the records do not even include pictures of the items, let alone links to the three-dimensional models, and the records that do include pictures do not necessarily include such links. Even when a record has a link to the three-dimensional model, it actually redirects to the full list of models rather than to the individual item. There is no search functionality from the initial list of three-dimensional models, and no way to browse other than to, colloquially speaking, poke and hope. Individual items are only identified by item number, and, aside from the few records that have accompanying pictures on the University of Birmingham site, there is no way to know to what item any given number refers. The 
website attains only a low rating for content; although it seems that there may be a decent number of items in the collection, it is impossible to know for certain given the problems with the interface and the fact that individual items are virtually unidentified.

The Eton Myers Collection website also receives a low rating for functionality. In order to access three-dimensional models of items, users must download and install a program called MeshLab, then download individual folders of compressed files, then unzip those files, and finally open the appropriate file in MeshLab. Despite compression, some of the file folders are still quite large and take some time to download. Presentation of the items is also rated low. Even for the high resolution versions of the three-dimensional renderings, viewed in MeshLab, the geometry of the objects seems underdeveloped (e.g., hieroglyphics are illegible) and surface textures are not well mapped (e.g., colors are completely off). This is evident from a comparison of the threedimensional rendering with a two-dimensional photograph of the same item, as in figure 2 , below.

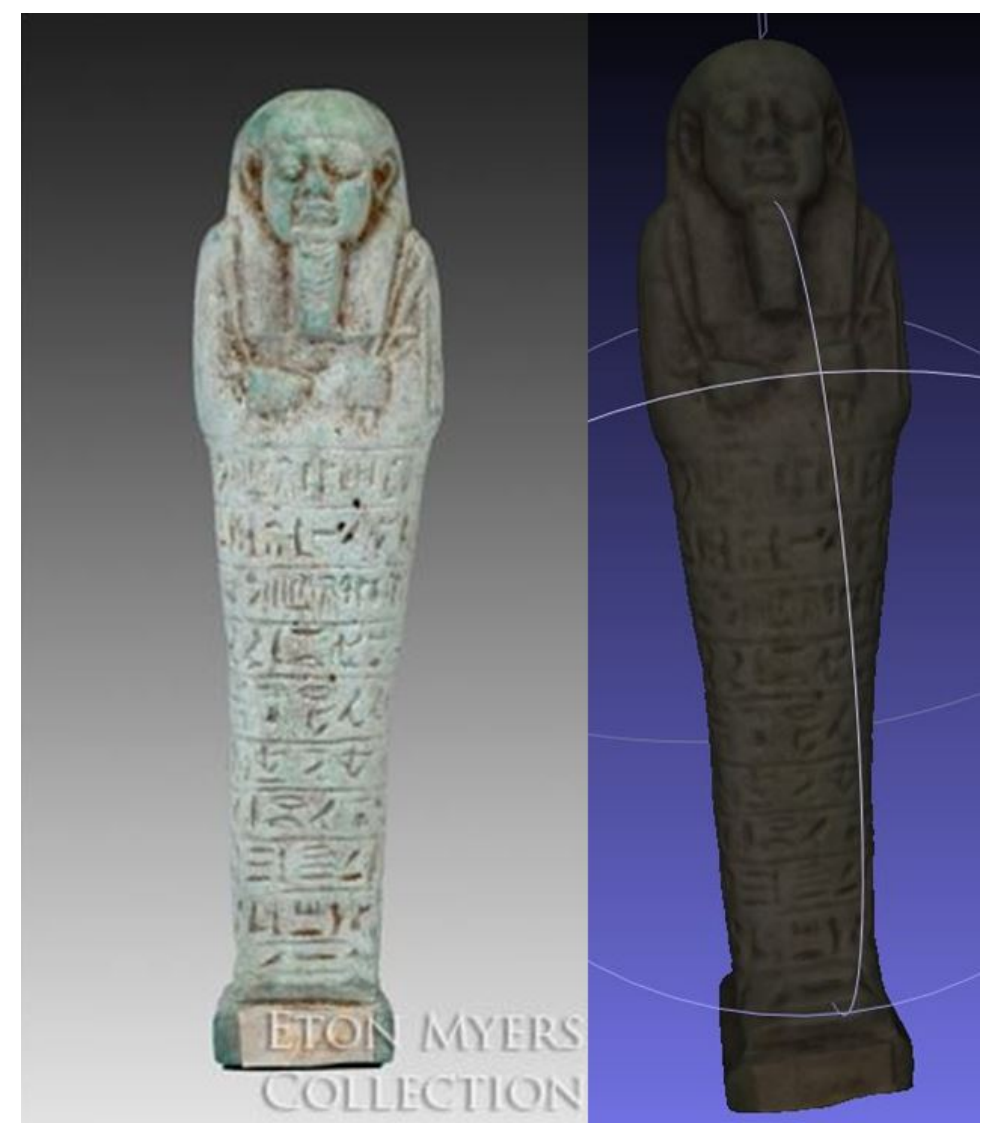

Figure 2. Comparison of original photograph (left) and three-dimensional rendering (right) of Item Number ECM 361, from the Eton Myers Collection

(http://mimsy.bham.ac.uk/detail.php?t=objects\&type=ext\&f=\&s=\&record=0\&id number=ecm +3 61\&op-earliest year=\%3D\&op-latest year=\%3D).

Notably, Chapman, Gaffney, and Moulden (2010) indicate that the detailed three-dimensional imaging enabled them to identify tooling marks and read previously unclear hieroglyphics on certain items. Thus, it is possible that the problems with the renderings may be a result of a loss in quality between the original models and the downloaded versions, particularly given that the files were reduced in size and converted prior to being made available for download. 


\section{Epigraphia 3D Project (http://www.epigraphia3d.es)}

The Epigraphia 3D project was created to present an online collection of various historical Roman epigraphs (also known as inscriptions) that were discovered and excavated in Spain and Italy; the physical collection is housed at the Museo Arqueológico Nacional (Madrid). Digital imaging was accomplished using photogrammetry, free software was utilized to create three-dimensional object models and renderings, and Photoshop was used to obtain appropriate textures. Finally, the three-dimensional model was published on the web using Sketchfab, a web service similar to Flickr that allows in-browser viewing of three-dimensional renderings in many different formats (Ramírez-Sánchez et al. 2014).

The Epigraphia 3D website is intuitive and informative. Browsing is simple because there are not many records, but, although it is possible to search the website, there is no search function specifically directed to the collection. Thus, usability is rated as medium. Despite the fact that the website provides descriptions of the project and the collection, as well as information about epigraphs generally, the website attains a medium rating for content in light of the small size of the collection and the limited information given for each individual item. However, the Epigraphia 3D website receives very high ratings for functionality and presentation. The individual threedimensional models are detailed, legible, and interactive. Individual inscriptions are transcribed for each item. The use of Sketchfab to display the models is effective; no downloading is necessary, and it takes an acceptable amount of time to load. When viewing the item, users can rotate the object in either "orbit" or "first person" mode, as well as view it full-screen or within the browser window. Users can also display the wireframe model and the textured or surfaced rendering, as shown in Figure 3 below.

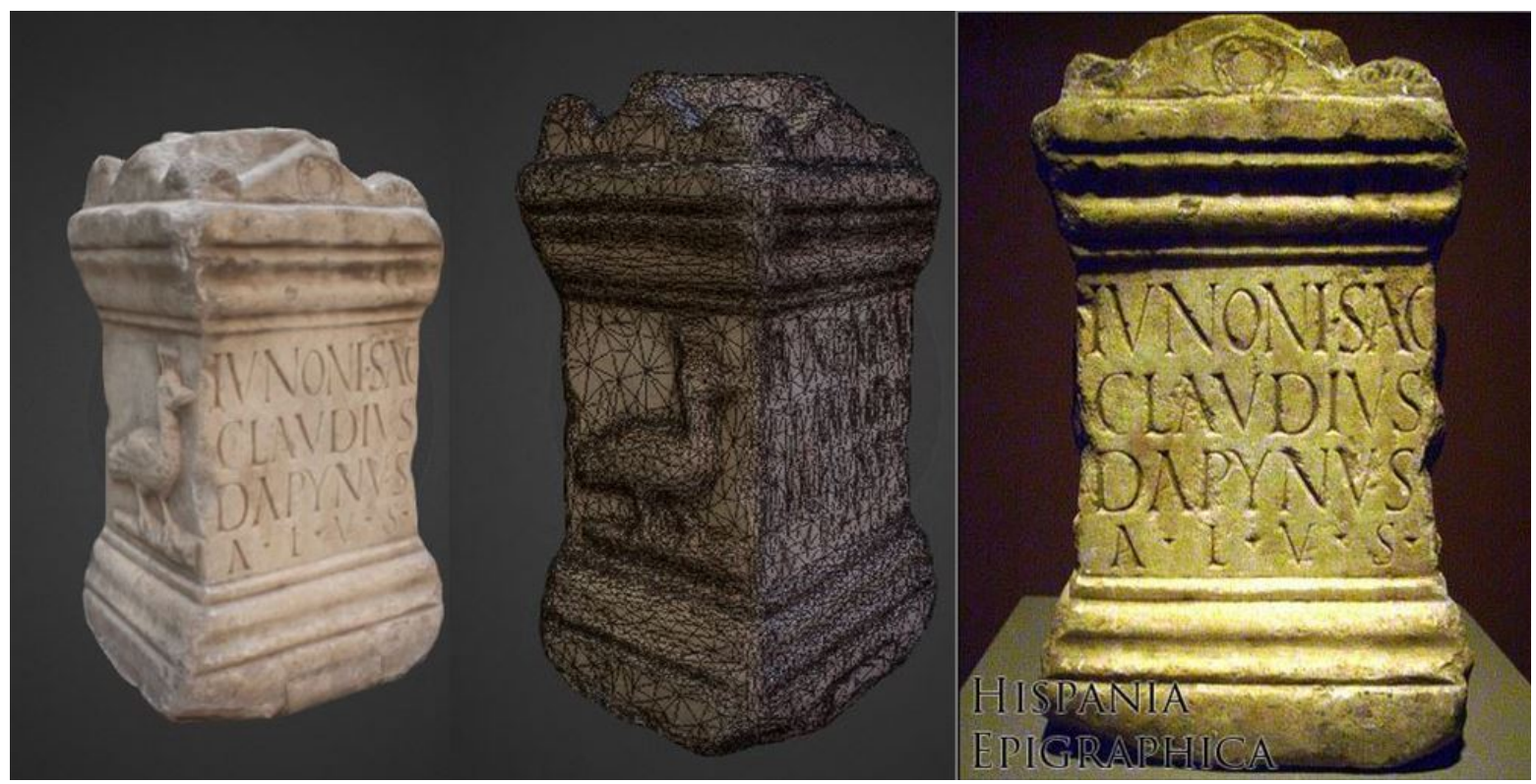

Figure 3. Three-dimensional textured (left) and wireframe (middle) renderings from the Epigraphia 3D project (http://www.epigraphia3d.es/3d-01.html), as compared to an original twodimensional photograph of the same object (right) (http://edabea.es/pub/record card 1.php?refpage=\%2Fpub\%2Fsearch select.php\&quicksearch=dapynus\&r

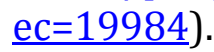




\section{Smithsonian X 3D (http://3d.si.edu)}

The Smithsonian X 3D project, although affiliated with all of the Smithsonian's varying divisions, was created to test the application of three-dimensional digitization techniques to "iconic collection objects" (http://3d.si.edu/about). The website provides significant detail concerning the project itself, mostly in the form of videos, and individual items, many of which are linked to "tours" that incorporate a story about the object. Content is rated as medium because, despite the depth of information provided about individual items, there are still very few items within the collection. The website also receives a medium rating for usability, given the simple browsing structure, easy navigation, and lack of a search feature (all likely due at least in part to the limited content). Functionality and presentation, however, are rated high. The X3D Explorer in-browser software (powered by Autodesk) does more than simply display a three-dimensional rendering of an object; it also permits users to edit the model by changing color, lighting, texture, and other variables as well as incorporates detailed information about each item, both as an overall description and as a slide show, where snippets of information are connected to specific views of the item. The individual three-dimensional models are high resolution, detailed, and wellrendered, with very good surface texture mapping. However, it must be noted that the X3D Explorer tool is in Beta and, as such, still has some bugs; for example, this author has observed a model disappear while zooming in on the rendering.

Table 3, below, summarizes the results of the evaluation.

\begin{tabular}{lllll}
\hline & Functionality & Usability & Presentation & Content \\
\hline MFA & Medium & Very High & Low & Very High \\
DFL & Medium & High & Medium & High \\
Eton Myers & Low & Low & Low & Low \\
Epigraphia 3D & Very High & Medium & Very High & Medium \\
Smithsonian X 3D & High & Medium & High & Medium \\
\hline
\end{tabular}

Table 3. Summary of evaluation results for each website by individual criteria

\section{DISCUSSION}

Based on the evaluation of the five websites described above, some suggested best practices for the digitization and presentation of three-dimensional objects become apparent. When digitizing, the museum should utilize the method that best suits the object or collection. For example, while MRI scanning is likely the best method for three-dimensionally digitizing biological fish specimens, it is not going to be effective or feasible for digitizing artwork or artifacts (Abel et al. 2011; Berquist et al. 2012). Regardless of the method of digitization used, however, the people conducting the imaging and processing should fully comprehend the hardware and software necessary to complete the task.

Additionally, although financial restraints must be considered, museums should note that some three-dimensional scanning equipment is just as economically feasible as standard digital cameras (Metallo and Rossi 2011). However, if a museum chooses to utilize only two-dimensional imaging, 
each item should be photographed from multiple angles in high resolution, to avoid creating a website, like the MFA's, on which everything other than the object itself is presented outstandingly. Further, museums deciding on two-dimensional imaging should explore the possibility of utilizing photogrammetry to create three-dimensional models from their twodimensional photographs, like the Epigraphia 3D project. There is free or inexpensive software that functions to permit the creation of three-dimensional object maps from very few photographs (Ramírez-Sánchez et al. 2014). Finally, compatibility is a key issue when conducting threedimensional scans; the museum should ensure that the software used for rendering models is compatible with the way in which users will be viewing the models.

In the context of public access to the museum's digital collections, the website should be easy and intuitive to navigate. The MFA website is an excellent example; browsing and search functions should both be present, and reorganization of large numbers of objects into separate collections may be necessary. Where searching is going to be the primary point of entry into the collection, it is important to have sufficient metadata and functional search algorithms to ensure that item records are findable.

Furthermore, remember that the website is simply a way to access the museum itself. Hence, the collections on the website, like the collections in the physical museum, should be curated; there should be a logical flow to accessing object records. The museum may also want to have sections that are similar to virtual exhibitions, like the "tours" provided by the Smithsonian X 3D project. Finally, museums should ensure that no additional technological know-how (beyond being able to access the internet) is required to access the three-dimensional content in object records. Users should not be required to download software or files to view records; Epigraphia 3D's use of Sketchfab and the Smithsonian's X 3D Explorer tool are both excellent examples of ways in which three-dimensional content can be viewed on the web without the need for extraneous software.

Museums and cultural heritage institutions are increasing the focus on providing public access to collections via digitization and display on websites (Given and McTavish 2010). In order to do this effectively, this paper has attempted to provide some guidance as to best practices of presenting digital versions of three-dimensional objects. In closing, however, it must be noted that this author is not a technician. Although this paper has tried to contend with the issues from the perspective of a librarian, there are complicated technical concerns behind any digitization project that have not been adequately addressed. In addition, this paper has not examined the role of budgetary constraints on digitization or the concomitant issues of creating and maintaining websites. Moreover, because this paper has been treated as a broad overview of the digitization and presentation for public access of three-dimensional objects, the five websites evaluated were from varying fields of study. Museums should look to more specific comparisons in order to appropriately digitize and present their collections on the web.

\section{CONCLUSION}

There may not be a direct substitute for encountering an object in person, but for people who cannot obtain physical access to three-dimensional objects, the digital realm can serve as an adequate proxy. This paper has demonstrated, through an evaluation of five distinct digital collections, that utilizing three-dimensional imaging and presenting three-dimensional models of physical objects on the web can serve the important purpose of increasing public access to otherwise unavailable collections. 


\section{REFERENCES}

Abate, D., R. Ciavarella, G. Furini, G. Guarnieri, S. Migliori, and S. Pierattini. "3D Modeling and Remote Rendering Technique of a High Definition Cultural Heritage Artefact." Procedia Computer Science 3 (2011): 848-52. http://dx.doi.org/10.1016/j.procs.2010.12.139.

Abel, R. L., S. Parfitt, N. Ashton, Simon G. Lewis, Beccy Scott, and C. Stringer. "Digital Preservation and Dissemination of Ancient Lithic Technology with Modern Micro-CT." Computers and Graphics 35, no. 4 (August 2011): 878-84. http://dx.doi.org/10.1016/j.cag.2011.03.001.

Berquist, Rachel M., Kristen M. Gledhill, Matthew W. Peterson, Allyson H. Doan, Gregory T. Baxter, Kara E. Yopak, Ning Kang, H.J. Walker, Philip A. Hastings, and Lawrence R. Frank. "The Digital Fish Library: Using MRI to Digitize, Database, and Document the Morphological Diversity of Fish." PLOS ONE 7, no. 4: (April 2012). http://dx.doi.org/10.1371/journal.pone.0034499.

Bincsik, Monika, Shinya Maezaki, and Kenji Hattori. "Digital Archive Project to Catalogue Exported Japanese Decorative Arts." International Journal of Humanities and Arts Computing 6, no. 12 (March 2012): 42-56. http://dx.doi.org/10.3366/ijhac.2012.0037.

Cameron, Fiona. "Digital Futures I: Museum Collections, Digital Technologies, and the Cultural Construction of Knowledge." Curator: The Museum Journal 46, no. 3 (July 2003): 325-40. http://dx.doi.org/10.1111/j.2151-6952.2003.tb00098.x.

Chane, Camille Simon, Alamin Mansouri, Franck S. Marzani, and Frank Boochs. "Integration of 3D and Multispectral Data for Cultural Heritage Applications: Survey and Perspectives." Image and Vision Computing 31, no. 1 (January 2013): 91-102. http://dx.doi.org/10.1016/j.imavis.2012.10.006.

Chapman, Henry P., Vincent L. Gaffney, and Helen L. Moulden. "The Eton Myers Collection Virtual Museum." International Journal of Humanities and Arts Computing 4, no. 1-2 (October 2010): 81-93. http://dx.doi.org/10.3366/ijhac.2011.0009.

Dellepiane, M., M. Callieri, F. Ponchio, and R. Scopigno. "Mapping Highly Detailed Colour Information on Extremely Dense 3D Models: The Case of David's restoration." Computer Graphics Forum 27, no. 8 (December 2008): 2178-87. http://dx.doi.org/10.1111/i.14678659.2008.01194.X.

Given, Lisa M., and Lianne McTavish. "What's Old Is New Again: The Reconvergence of Libraries, Archives, and Museums in the Digital Age." Library Quarterly 80, no. 1 (January 2010): 732. http://dx.doi.org/10.1086/648461.

Hariri, Nadjla, and Yaghoub Norouzi. “Determining Evaluation Criteria for Digital Libraries' User Interface: A Review." The Electronic Library 29, no. 5 (2011): 698-722. http://dx.doi.org/10.1108/02640471111177116.

Hess, Mona, Francesca Simon Millar, Stuart Robson, Sally MacDonald, Graeme Were, and Ian Brown. "Well Connected to Your Digital Object? E-curator: A Web-Based E-Science Platform for Museum Artefacts." Literary and Linguistic Computing 26, no. 2 (2011): 193 215. http://dx.doi.org/10.1093/llc/fqr006. 
Holovachov, Oleksandr, Andriy Zatushevsky, and Ihor Shydlovsky. "Whole-Drawer Imaging of Entomological Collections: Benefits, Limitations and Alternative Applications." Journal of Conservation and Museum Studies 12, no. 1 (2014): 1-13.

http://dx.doi.org/10.5334/jcms.1021218.

Hunter, Jane, and Anna Gerber. 2010. "Harvesting Community Annotations on 3D Models of Museum Artefacts to Enhance Knowledge, Discovery and Re-Use." Journal of Cultural Heritage 11, no. 1 (2010): 81-90. http://dx.doi.org/10.1016/j.culher.2009.04.004.

Jarrell, Michael C. "Providing Access to Three-Dimensional Collections." Reference \& User Services Quarterly 38, no. 1 (1998): 29-32.

Kravchyna, Victoria, and Sam K. Hastings. "Informational Value of Museum Web Sites." First Monday 7, no. 4 (February 2002). http://dx.doi.org/10.5210/fm.v7i2.929.

Kuzminsky, Susan C. and Megan S. Gardiner. "Three-Dimensional Laser Scanning: Potential Uses for Museum Conservation and Scientific Research." Journal of Archaeological Science 39, no. 8 (August 2012): 2744-51. http://dx.doi.org/10.1016/i.jas.2012.04.020.

Lerma, José Luis, and Colin Muir. "Evaluating the 3D Documentation of an Early Christian Upright Stone with Carvings from Scotland with Multiples Images." Journal of Archaeological Science 46 (June 2014): 311-18. http://dx.doi.org/10.1016/i.jas.2014.02.026.

Louw, Marti, and Kevin Crowley. "New Ways of Looking and Learning in Natural History Museums: The Use of Gigapixel Imaging to Bring Science and Publics Together." Curator: The Museum Journal 56, no. 1 (January 2013): 87-104. http://dx.doi.org/10.1111/cura.12009.

Metallo, Adam, and Vince Rossi. "The Future of Three-Dimensional Imaging and Museum Applications." Curator: The Museum Journal 54, no. 1 (January 2011): 63-69. http://dx.doi.org/10.1111/i.2151-6952.2010.00067.x.

Montani, Isabelle, Eric Sapin, Richard Sylvestre, and Raymond Marquis . "Analysis of Roman Pottery Graffiti by High Resolution Capture and 3D Laser Profilometry." Journal of Archaeological Science 39, no. 11 (2012): 3349-53. http://dx.doi.org/10.1016/i.jas.2012.06.011.

Newell, Jenny. "Old Objects, New Media: Historical Collections, Digitization and Affect." Journal of Material Culture 17, no. 3 (September 2012): 287-306. http://dx.doi.org/10.1177/1359183512453534.

Novati, Gianluca, Paolo Pellegri, and Raimondo Schettini. "An Affordable Multispectral Imaging System for the Digital Museum." International Journal on Digital Libraries 5, no. 3 (May 2005): 167-78. http://dx.doi.org/10.1007/s00799-004-0103-y.

Pallas, John, and Anastasios A. Economides. "Evaluation of Art Museums' Web Sites Worldwide." Information Services and Use 28, no. 1 (2008): 45-57. http://dx.doi.org/10.3233/ISU2008-0554.

Parandjuk, Joanne C. "Using Information Architecture to Evaluate Digital Libraries." The Reference Librarian 51, no. 2 (2010): 124-34. http://dx.doi.org/10.1080/02763870903579737. 
Pavlidis, George, Anestis Koutsoudis, Fotis Arnaoutoglou, Vassilios Tsioukas, and Christodoulos Chamzas. "Methods for 3D Digitization of Cultural Heritage." Journal of Cultural Heritage 8, no. 1 (2007): 93-98, http://dx.doi.org/10.1016/j.culher.2006.10.007.

Ramírez-Sánchez, Manuel, José-Pablo Suárez-Rivero, and María-Ángeles Castellano-Hernández. "Epigrafía digital: tecnología 3D de bajo coste para la digitalización de inscripciones y su acceso desde ordenadores y dispositivos móviles." El Profesional de la Información 23, no. 5 (2014): 467-74. http://dx.doi.org/10.3145/epi.2014.sep.03.

Saracevic, Tefko. "Digital Library Evaluation: Toward an Evolution of Concepts." Library Trends 49 , no. 3 (2000): 350-69.

Srinivasan, Ramesh, Robin Boast, Jonathan Furner, and Katherine M. Becvar. "Digital Museums and Diverse Cultural Knowledges: Moving past the Traditional Catalog." The Information Society 25, no. 4 (2009): 265-78, http://dx.doi.org/10.1080/01972240903028714.

Xie, Hong Iris. "Users' Evaluation of Digital Libraries (DLs): Their Uses, Their Criteria, and Their Assessment." Information Processing and Management 44, no. 3 (May 2008): 1346-73, http://dx.doi.org/10.1016/j.ipm.2007.10.003. 